OPEN ACCESS

Edited by:

Baolei Jia,

Chung-Ang University, South Korea

Reviewed by: Joshua Chang Mell, Drexel University, United States

Sunil Kumar Verma,

The University of Texas Medical Branch at Galveston, United States

${ }^{*}$ Correspondence:

Anchun Cheng

chenganchun@vip.163.com

†These authors have contributed equally to this work

Specialty section:

This article was submitted to Evolutionary and Genomic Microbiology,

a section of the journal Frontiers in Genetics

Received: 24 January 2019 Accepted: 18 April 2019

Published: 17 May 2019

Citation:

Huang L, Tian X, Liu M, Wang M, Biville $F$, Cheng $A$, Zhu D, Jia $R$,

Chen S, Zhao $X$, Yang $Q$, Wu Y,

Zhang S, Huang J, Tian B, Yu Y, Liu Y, Zhang L, Pan L, Rehman MU and

Chen X (2019) DprA is Essential for Natural Competence in Riemerella anatipestifer and Has a Conserved

Evolutionary Mechanism Front. Genet. 10:429.

doi: 10.3389/fgene.2019.00429

\section{DprA Is Essential for Natural Competence in Riemerella anatipestifer and Has a Conserved Evolutionary Mechanism}

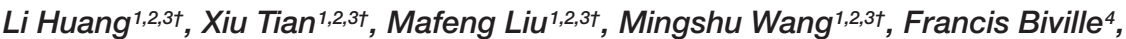 \\ Anchun Cheng ${ }^{1,2,3 *}$, Dekang Zhu ${ }^{2,3}$, Renyong Jia ${ }^{1,2,3}$, Shun Chen ${ }^{1,2,3}$, Xinxin Zhao ${ }^{1,2,3}$, \\ Qiao Yang 1,2,3, Ying Wu ${ }^{1,2,3}$, Shaqiu Zhang ${ }^{1,2,3}$, Juan Huang ${ }^{1,2,3}$, Bin Tian 1,2,3, Yanling Y $\mathbf{u}^{2,3}$, \\ Yunya Liu ${ }^{1,2,3}$, Ling Zhang ${ }^{2,3}$, Leichang Pan ${ }^{1,2,3}$, Mujeeb Ur Rehman ${ }^{1,2,3}$ and \\ Xiaoyue Chen ${ }^{2,3}$

\begin{abstract}
${ }^{1}$ Institute of Preventive Veterinary Medicine, Sichuan Agricultural University, Chengdu, China, ${ }^{2}$ Research Center of Avian Disease, College of Veterinary Medicine, Sichuan Agricultural University, Chengdu, China, ${ }^{3}$ Key Laboratory of Animal Disease and Human Health of Sichuan Province, College of Veterinary Medicine, Sichuan Agricultural University, Chengdu, China, ${ }^{4}$ Institut Pasteur, Paris, France
\end{abstract}

Riemerella anatipestifer ATCC11845 (RA ATCC11845) is naturally competent. However, the genes involved in natural transformation in this species remain largely unknown. Bioinformatic analysis predicts that DprA of RA (DprA $\left.A_{R a}\right)$ has three domains: a sterile alpha motif (SAM), a Rossmann fold (RF) domain and a Z-DNA-binding domain (Z $\alpha)$. Inactivation of dprA abrogated natural transformation in RA ATCC11845, and this effect was restored by the expression of dprA in trans. The dprA with SAM and RF domains of Streptococcus pneumoniae and the dprA with RF and Z $\alpha$ domains of Helicobacter pylori was able to restore natural transformation in the RA ATCC11845 dprA mutant. An Arg123 mutation in the RF domain of $R$. anatipestifer was not able to restore natural transformation of the RA ATCC11845 dprA mutant. Furthermore, DprA ${ }^{\text {R123E }}$ abolished its ability to bind DNA, suggesting that the RF domain is essential for the function of DprA. Finally, the dprA of Fusobacterium naviforme which has not been reported to be natural competent currently was partially able to restore natural transformation in RA ATCC11845 dprA mutant. These results collectively suggest that DprA has a conserved evolutionary mechanism.

Keywords: Riemerella anatipestifer, dprA, natural competence, EMSA, evolution

\section{INTRODUCTION}

Natural transformation involves the acquisition of naked DNA by a bacterium from the extracellular environment and integrated DNA into their genome, and genetic competence is the ability to undergo natural transformation (Chen and Dubnau, 2004). At present, at least 82 bacterial species have been shown to be naturally transformable (Johnston et al., 2014; Mell and Redfield, 2014). The process of natural transformation involves two steps: DNA uptake and DNA processing (Johnston et al., 2014). Gram-positive and Gram-negative bacteria rely on highly similar DNA-uptake systems (Johnston et al., 2014). Transformable Gram-negative species use proteins 
that are related to those involved in the assembly of type II secretion systems and type IV pili (T4P) (Hobbs and Mattick, 1993; Dubnau, 1999). It has been proposed that structure similar to T4P known as a competence pseudopilus participates in DNA transport in Gram-positive bacteria (Dubnau, 1999). Once dsDNA has entered the periplasm, one strand of the dsDNA is degraded, and the other strand is internalized via a ComEC transmembrane channel (Draskovic and Dubnau, 2005). After internalization, the transforming ssDNA is bound to the transformation-dedicated recombination mediator protein (RMP) DprA, which loads the homologous recombinase RecA to promote homologous recombination (Mortier-Barriere et al., 2007; Yadav et al., 2013).

Riemerella anatipestifer ( $R$. anatipestifer, RA) is a Gramnegative bacterium that causes septicemic diseases in ducks, geese, turkeys, and other birds (Huang et al., 2017). At present, at least 21 serotypes of $R$. anatipestifer have been identified (reference). The extensive use of antibiotics for the treatment and prevention of serositis and septicemia has resulted in multidrug resistance in $R$. anatipestifer (Zhong et al., 2009; Luo et al., 2015, 2018; Huang et al., 2017; Zhang et al., 2017; Zhu et al., 2018), and at least 33 relevant bacterial genomes with genome sizes ranging from 2.09 to $2.43 \mathrm{Mb}$ have been sequenced from different isolates (Wang X. et al., 2014; Song et al., 2016; Zhu et al., 2016). Sequence analysis of RA ATCC11845, RA CH-1, and RA CH-2 showed that RA CH-1 is 140,000 bp larger than the two other strains and there are so many resistance genes in this region (Wang $\mathrm{X}$. et al., 2014). However, the reason for this genome diversity and the mechanisms underlying multidrug resistance remain largely unknown. In our previous study, we showed that $R$. anatipestifer is naturally competent (Liu et al., 2017). At present, the machinery involved in the taking up of exogenous dsDNA involves T4P or T4SS (Johnston et al., 2014). However, no homologs of T4P and T4SS were found in the genome of $R$. anatipestifer through sequence comparison. Several genes which was annotated as $\operatorname{rec} A, \operatorname{dpr} A, \operatorname{com} M$, and $\operatorname{comEC}$, respectively, in the genome of $R$. anatipestifer are predicted to be involved in transporting DNA across inner membrane and homologous recombination (Liu et al., 2017). Bioinformatic analysis showed that the gene RA0C_1073 of RA ATCC11845 encodes a putative DprA with low identity to the DprA found in other bacteria. In addition, RA ATCC11845 is the first bacteria in Flavobacteriaceae that has been reported to have natural competence. In this study, we sought to provide a functional characterization of DprA and its mechanism in the natural transformation of RA ATCC11845. This information will be helpful for determining the mechanism of natural transformation in bacteria occurring in $R$. anatipestifer and other members of Flavobacteriaceae.

\section{MATERIALS AND METHODS}

\section{Bacterial Strains, Plasmids and Primers}

The bacterial strains and plasmids used in this study are shown in Table 1. The primers are shown in Table 2.

\section{Media and Growth Conditions}

Riemerella anatipestifer was routinely cultured in GC broth (GCB) with agitation (Liu et al., 2017) or on LB plates supplemented with $5 \%$ sheep blood at $37^{\circ} \mathrm{C}$ (Liu et al., 2018a). GCB agar plates were prepared by using GCB supplemented with $1.5 \%$ agar. Escherichia coli strains were grown on LB agar at $37^{\circ} \mathrm{C}$. When required, antibiotics were added at the following final concentrations ( $\mu \mathrm{g} / \mathrm{ml})$ : erythromycin (Erm), 1; cefoxitin (Cfx), 1 ; and streptomycin (Str), 100 for $R$. anatipestifer and ampicillin (Amp), 100 for E. coli.

\section{Natural Transformation}

Natural transformation was performed as described previously with slight modification (Liu et al., 2017). Briefly, the single colony was isolated on LB agar supplemented with $5 \%$ sheep blood at $37^{\circ} \mathrm{C}$ for $24 \mathrm{~h}$. The eugonic cells were collected from the plate into GCB medium (Liu et al., 2017) and checked for OD600. Then, the bacteria were adjusted to an OD600 of 1 . The bacterial suspensions $(0.3 \mathrm{ml})$ were transferred to sterilized tubes, and $1 \mu \mathrm{g}$ of plasmid DNA or genomic DNA was added to the tube. After an additional incubation for $1 \mathrm{~h}$ at $37^{\circ} \mathrm{C}$, the bacterial cultures were serially diluted and plated onto GCB agar plates or LB agar supplemented with $5 \%$ sheep blood with or without antibiotics. Transformation frequencies were calculated as the CFU ml ${ }^{-1}$ on selective plates divided by the CFU ml $\mathrm{ml}^{-1}$ on non-selective plates (Kristensen et al., 2012).

\section{Construction of the RA ATCC11845 dprA Mutant}

The RA ATCC11845 $d p r A$ mutant was constructed using the natural transformation method as described previously (Liu et al., 2017). Briefly, the upstream sequence ( 620 bp) and the downstream sequence $(\sim 620 \mathrm{bp})$ of the $d p r A$ gene were amplified using the primers dprA upP1 and dprA upP2 or dprA downP1 and dprA downP2, respectively (Table 2). The 994-bp ErmR cassette was amplified from the RA-CH-1 strain (Luo et al., 2015) (Liao plos one) using the primers ErmP1 and ErmP2 (Table 2). The resulting PCR fragments were ligated using the overlapped PCR method (Xiong et al., 2006). The fused PCR fragments were purified by TianGEN (TIANGEN, Beijing, China) and introduced into the wild-type strain by natural transformation. The transformants were selected on blood agar plates supplemented with Erm $(1 \mu \mathrm{g} / \mathrm{ml})$. The gene-deletion mutant strain was identified by PCR by amplifying the conserved 16S rRNA gene of $R$. anatipestifer using the primers 16S rRNAP1 and $16 \mathrm{~S}$ rRNAP2 to verify whether the mutant is $R$. anatipestifer, and the $d p r A$ gene was amplified using the primers dprA Comp P1 and dprA Comp P2.

\section{Preparation of Transformation DNA (tDNA)}

Streptomycin-resistant RA ATCC11845 cells were obtained by plating $10^{8}$ wild-type cells on LB agar supplemented with 5\% sheep blood containing $100 \mu \mathrm{g} / \mathrm{ml}$ streptomycin. Streptomycinresistant clones were streaked for isolation on fresh medium, and the rpsL of each clone was PCR-amplified using the primers 
TABLE 1 | Strains and plasmids used in this study.

\begin{tabular}{|c|c|c|}
\hline Riemerella anatipestifer strains & Genotype or description & $\begin{array}{l}\text { Source or } \\
\text { references }\end{array}$ \\
\hline RA ATCC11845 & RA ATCC11845, $\mathrm{Km}^{\mathrm{R}}$ & $\begin{array}{l}\text { Laboratory } \\
\text { collection }\end{array}$ \\
\hline RA ATCC11845 $\Delta d p r A:: E r m$ & RA ATCC11845 $\Delta d p r A, \mathrm{Erm}^{\mathrm{R}}$ & This study \\
\hline RA ATCC11845 (pLMF03::dprA) & RA ATCC11845 carrying pLMF03::dprA, Amp ${ }^{R}$ Cfx ${ }^{R}$ & This study \\
\hline RA ATCC11845 (pLMF03::dprA R123E) & RA ATCC11845 carrying pLMF03::dprA ${ }^{R 123 E}$, Amp $^{R}$ Cfx ${ }^{R}$ & This study \\
\hline RA ATCC11845 (pLMF03::dprA-SAM-RF) & RA ATCC11845 carrying pLMF03::dprA-SAM-RF, Amp ${ }^{R}$ Cfx ${ }^{R}$ & This study \\
\hline RA ATCC11845 (pLMF03::dprA-RF-Z $\alpha$ ) & RA ATCC11845 carrying pLMF03::dprA-RF-Z $\alpha$, Amp $^{R}$ Cfx ${ }^{R}$ & This study \\
\hline RA ATCC11845 (pLMF03::Sp-dprA) & RA ATCC11845 carrying pLMF03::Sp-dprA, Amp ${ }^{R}$ Cfx ${ }^{R}$ & This study \\
\hline RA ATCC11845 (pLMF03::Hp-dprA) & RA ATCC11845 carrying pLMF03::Hp-dprA, Amp ${ }^{R}$ Cfx ${ }^{R}$ & This study \\
\hline RA ATCC11845 (pLMF03::Fn-dprA) & RA ATCC11845 carrying pLMF03::Fn-dprA, Amp ${ }^{R}$ Cfx $^{R}$ & This study \\
\hline RA ATCC11845 $\Delta d p r A:: E r m$ (pLMF03::dprA) & RA ATCC11845 $\Delta d p r A$ carrying pLMF03::dprA, Erm ${ }^{R}$ Amp $^{R}$ Cfx ${ }^{R}$ & This study \\
\hline RA ATCC11845 $\Delta d p r A:: E r m$ (pLMF03::dprA $\left.{ }^{\mathrm{R} 123 \mathrm{E}}\right)$ & RA ATCC11845 $\Delta d p r A$ carrying pLMF03::dprA ${ }^{R 123 E}$, Erm $^{R} A_{m p}^{R} C f x^{R}$ & This study \\
\hline RA ATCC11845 $\Delta d p r A:: E r m$ (pLMF03::dprA-SAM-RF) & RA ATCC11845 $\Delta d p r A$ carrying pLMF03::dprA-SAM-RF, Erm ${ }^{R}$ Amp $^{R}$ Cfx ${ }^{R}$ & This study \\
\hline RA ATCC11845 $\Delta d p r A:: E r m(p L M F 03:: d p r A-R F-Z \alpha)$ & RA ATCC11845 $\Delta d p r A$ carrying pLMF03::dprA-RF-Z $\alpha$, Erm $^{R}$ Amp $^{R}$ Cfx $^{R}$ & This study \\
\hline RA ATCC11845 $\Delta d p r A:: E r m(p L M F 03:: S p-d p r A)$ & RA ATCC11845 $\Delta d p r A$ carrying pLMF03::Sp-dprA, Erm ${ }^{R} \mathrm{Amp}^{\mathrm{R}} \mathrm{Cfx}{ }^{\mathrm{R}}$ & This study \\
\hline RA ATCC11845 $\Delta d p r A:: E r m(p L M F 03:: H p-d p r A)$ & RA ATCC11845 $\Delta d p r A$ carrying pLMF03::Hp-dprA, Erm Amp $^{R} \mathrm{Cfx}^{\mathrm{R}}$ & This study \\
\hline RA ATCC11845 $\Delta d p r A:: E r m(p L M F 03:: F n-d p r A)$ & RA ATCC11845 $\Delta d p r A$ carrying pLMF03::Fn-dprA, Erm Amp $^{R}$ Cfx ${ }^{R}$ & This study \\
\hline Escherichia coli strains & Genotype or description & $\begin{array}{l}\text { Source or } \\
\text { references }\end{array}$ \\
\hline $\mathrm{DH} 5 \alpha$ & E. coli $\mathrm{DH} 5 \alpha$, cloning host cell & $\begin{array}{l}\text { Laboratory } \\
\text { collection }\end{array}$ \\
\hline XL1-blue & E. coli XL1-blue, cloning host cell & $\begin{array}{l}\text { Laboratory } \\
\text { collection }\end{array}$ \\
\hline Rosetta & E. coli Rosetta, expressing host cell & $\begin{array}{l}\text { Laboratory } \\
\text { collection }\end{array}$ \\
\hline Rosetta (pET30a) & E. coli Rosetta carrying pET30a, $\mathrm{Km}^{\mathrm{R}}$ & This study \\
\hline Rosetta (pET30a::dprA-s) & E. coli Rosetta carrying pET30a::dprA, $\mathrm{Km}^{\mathrm{R}}$ & This study \\
\hline Rosetta (pET30a::dprA $\left.{ }^{\mathrm{R} 123 \mathrm{E}_{-s}}\right)$ & E. coli Rosetta carrying pET30a::dprA $\mathrm{R}^{\mathrm{R} 123 \mathrm{E}_{-} \mathrm{s}, \mathrm{Km}} \mathrm{m}^{\mathrm{R}}$ & This study \\
\hline $\mathrm{S} 17-1$ & Thi-1 thr leu tonA lac Y supE recA::RP4-2-Tc::Mu Km & $\begin{array}{l}\text { Miller and } \\
\text { Mekalanos, } \\
1988\end{array}$ \\
\hline S17-1 (pLMF03::dprA) & S17-1 carrying pLMF03::dprA, Amp ${ }^{R} \mathrm{Cfx}^{\mathrm{R}}$ & This study \\
\hline S17-1 (pLMF03::dprA $\left.{ }^{\mathrm{R} 123 \mathrm{E}}\right)$ & S17-1 carrying pLMF03::dprA ${ }^{R 123 E}, \mathrm{Amp}^{\mathrm{R}} \mathrm{Cfx}{ }^{\mathrm{R}}$ & This study \\
\hline S17-1 (pLMF03::dprA-SAM-RF) & S17-1 carrying pLMF03::dprA-SAM-RF, Amp ${ }^{R}$ Cfx ${ }^{R}$ & This study \\
\hline S17-1 (pLMF03::dprA-RF-Z $\alpha$ ) & S17-1 carrying pLMF03::dprA-RF-Z $\alpha, \mathrm{Amp}^{\mathrm{R}} \mathrm{Cfx}{ }^{\mathrm{R}}$ & This study \\
\hline S17-1 (pLMF03::Sp-dprA) & S17-1 carrying pLMF03::Sp-dprA, Amp ${ }^{R}$ Cfx ${ }^{R}$ & This study \\
\hline S17-1 (pLMF03::Hp-dprA) & S17-1 carrying pLMF03::Hp-dprA, Amp ${ }^{R} \mathrm{Cfx}^{\mathrm{R}}$ & This study \\
\hline S17-1 (pLMF03::Fn-dprA) & S17-1 carrying pLMF03::Fn-dprA, Amp ${ }^{R} \mathrm{Cfx}^{\mathrm{R}}$ & This study \\
\hline Plasmids & Genotype or description & $\begin{array}{l}\text { Source or } \\
\text { references }\end{array}$ \\
\hline pET30a & pBR322 lacZ, IPTG-inducible promoter, $\mathrm{Km}^{\mathrm{R}}$ & $\begin{array}{l}\text { Laboratory } \\
\text { collection }\end{array}$ \\
\hline pET30a::dprA-s & pET30a carrying dprA adding his tag from RA ATCC11845, $\mathrm{Km}^{\mathrm{R}}$ & This study \\
\hline pET30a::dprA ${ }^{\mathrm{R} 123 \mathrm{E}_{-s}}$ & pET30a carrying Arg123 site-directed mutant dprA adding his tag of RA ATCC11845, $\mathrm{Km}^{\mathrm{R}}$ & This study \\
\hline pLMF03 & B739_0921 promoter, oriColE1, ori pRA0726, Amp ${ }^{R}$ Cfx ${ }^{R}$ & Liu et al., 2016 \\
\hline pLMF03::dprA & pLMF03 carrying dprA from RA ATCC11845, Amp ${ }^{R}$ Cfx ${ }^{R}$ & This study \\
\hline pLMF03::dprA ${ }^{\mathrm{R} 123 \mathrm{E}}$ & pLMF03 carrying Arg123 site-directed mutant dprA of RA ATCC11845, Amp ${ }^{R}$ Cfx $^{R}$ & This study \\
\hline pLMF03::Sp-dprA & pLMF03 carrying dprA from S. pneumoniae, $\mathrm{Amp}^{\mathrm{R}} \mathrm{Cfx}{ }^{\mathrm{R}}$ & This study \\
\hline pLMF03::Hp-dprA & pLMF03 carrying dprA from H. pylori, Amp ${ }^{R} \mathrm{Cfx}^{\mathrm{R}}$ & This study \\
\hline pLMF03::Fn-dprA & pLMF03 carrying dprA from F. naviforme, Amp ${ }^{R}$ Cfx ${ }^{R}$ & This study \\
\hline pLMF03::dprA-SAM-RF & pLMF03 carrying SAM and RF domains of $d p r A$ from RA ATCC11845, Amp ${ }^{R} C f x^{R}$ & This study \\
\hline pLMF03::dprA-RF-Z $\alpha$ & pLMF03 carrying RF and $Z \alpha$ domains of $d p r A$ from RA ATCC11845, Amp $^{R} \mathrm{Cfx}^{\mathrm{R}}$ & This study \\
\hline
\end{tabular}

$A m p^{R}$, ampicillin resistance; $K m^{R}$, kanamycin resistance; $C f x^{R}$, cefoxitin resistance. 
TABLE 2 | Primers used in this study.

\begin{tabular}{|c|c|c|}
\hline Primers & Sequence & Organism \\
\hline 16SrRNAP1 & CGAAAGTGATAAGTTAGCCACCT & RA ATCC11845 \\
\hline 16SrRNAP2 & GCAGCACCTTGAAAATTGTCC & RA ATCC11845 \\
\hline dprA upP1 & ACAAGGGGTGGCTATGGCGGCAAGTC & RA ATCC11845 \\
\hline dprA upP2 & TAAGACTGGAAAGTGGTAACTAGCGCCTTGCCAT & RA ATCC11845 \\
\hline ErmP1 & GCAAGGCGCTAGTTACCACTITCCAGTCTTACG & RA ATCC11845 \\
\hline ErmP2 & 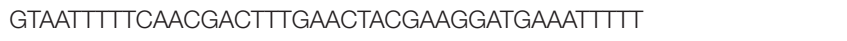 & RA ATCC11845 \\
\hline dprA downP1 & TCCTTCGTAGTTCAAAGTCGTTGAAAAATTACПIITAAAA & RA ATCC11845 \\
\hline dprA downP2 & TGCTTGGCAGAATCTCATAATITCCATATCCGA & RA ATCC11845 \\
\hline rpsLP1 & ATGCCTACTATTCAACAATTAG & RA ATCC11845 \\
\hline rpsLP2 & ПTACTППTAGCATCTITAGGACGC & RA ATCC11845 \\
\hline dprA CompP1 & CATGCCATGGCAATGGTAAATGCGGAAGAAATT & RA ATCC11845 \\
\hline dprA CompP2 & CCGCTCGAGCTAAATGATAGAATATCTCCTCCCA & RA ATCC11845 \\
\hline dprA-RFP1 & CATGCCATGGCAATGATTAAAAACGAAATAAAAAT & RA ATCC11845 \\
\hline dprA-RFP2 & CCGCTCGAGCTAAAAAAGCTCCAAAACTITAGA & RA ATCC11845 \\
\hline Sp-dprAP1 & CATGCCATGGCAATGGAGTTATITATGAAAATCACAA & S. pneumoniae D39 \\
\hline Sp-dprAP2 & CCGCTCGAGTTAAAATTCAAATTCCGCAAG & S. pneumoniae D39 \\
\hline Hp-dprAP1 & CATGCCATGGCAGTGAATCAACGAATGAAAAGCC & H. pylori 26695 \\
\hline Hp-dprAP2 & CCGCTCGAGTCACGCTAACACCACAATGTGA & H. pylori 26695 \\
\hline Fn-dprAP1 & CATGCCATGGCAATGGAGCTGACGAATCCACTTGG & synthesized \\
\hline Fn-dprAP2 & GCTCTAGAGCTTAAGGATGAAAGCGGGCACAG & synthesized \\
\hline dprA*P1 & AGTATTGTTGGGACGGAAAATGCCACTGCTTAT & RA ATCC11845 \\
\hline dprA*P2 & AGCAGTGGCATITTCCGTCCCAACAATACTAAT & RA ATCC11845 \\
\hline dprAExP1 & GGGAATTCCATATGGTAAATGCGGAAGAAATT & RA ATCC11845 \\
\hline dprAExP2his & CCGCTCGAGCTAGTGGTGGTGGTGGTGGTGAATGATAGAATATCTCCTCCCA & RA ATCC11845 \\
\hline RA-ssDNA 1 & TAGGCTCTGCTAAGGAAGCGTGGGGTCTGTCTAAGTTGGA & RA ATCC11845 \\
\hline RA-ssDNA 2 & AAAAACTACGGAACTGACTAAAGGCAGAAAAACTAAACGG & RA ATCC11845 \\
\hline EC-SsDNA & CTCAGGTGCGAAAGCGTGGGGAGCAAACAGGATTAGATAC & E. coli XL1-blue \\
\hline dprA qRTP1 & TCCGATGTITGAGGCAATTTG & RA ATCC11845 \\
\hline dprA qRTP2 & TGCAAGTITGGTTAGCGAGGTAG & RA ATCC11845 \\
\hline recA qRTP1 & CTTAGGATAACCGCCTACTC & RA ATCC11845 \\
\hline recA qRTP2 & CTTAGGATAACCGCCTACTC & RA ATCC11845 \\
\hline
\end{tabular}

rpslP1 and rpslP2 (Table 2) and then sent for sequencing. Point mutations in $r p s L$ conferring streptomycin resistance were identified by comparison to the wild type rpsL. Genomic DNA containing the rpsL mutant was extracted using the TIANamp Bacterial DNA Kit (TIANGEN, Beijing, China) and was used as the transformed DNA ( $\mathrm{tDNA}$ ). The concentration of the genomic DNA was measured by a Nanodrop 2000.

\section{Construction of the Recombinant Vectors for Complementation}

The insert fragment was cloned into the shuttle plasmid pLMF03, which contain high expression promoter, for complementation as described in previous study (Liu et al., 2016). Briefly, the entire coding region of $d p r A$ was amplified from RA ATCC11845 chromosomal DNA using the primers dprA Comp P1 and dprA Comp P2 (Table 2). The domain of $d p r A-S A M-R F$ and $d p r A-\mathrm{RF}-\mathrm{Z} \alpha$ for $d p r A$ was amplified from RA ATCC11845 chromosomal DNA using the primers dprA Comp P1 and dprA-RF P2, dprA-RF P1 and dprA Comp P2, respectively (Table 2). The entire coding region of Sp-dprA and Hp-dprA was amplified from the $S$. pneumoniae D39 strain and the
Helicobacter pylori 26695 strain using the primers Sp-dprAP1 and Sp-dprAP2, Hp-dprAP1 and Hp-dprAP2, respectively (Table 2). The entire coding region of Fn-dprA of Fusobacterium naviforme was synthesized according to the sequence (NCBI: EI53_RS03865) and amplified using the primers Fn-dprAP1 and Fn-dprAP2 (Table 2). The PCR products were purified, digested by the corresponding restriction endonuclease, and ligated into the plasmid pLMF03 to generate the plasmid pLMF03::dprA, pLMF03::dprA-SAM-RF, pLMF03::dprA-RF-Z $\alpha$, pLMF03::Sp-dprA, pLMF03::Hp-dprA, and pLMF03::Fn-dprA.

\section{Construction of a Site-Directed Mutant Recombination Vector}

Site-directed mutagenesis was carried out by overlap PCR to substitute the arginine residue (R) at position 123 of the R. anatipestifer $d p r A$ with glutamic acid (E). The desired mutant DNA fragment was obtained by two rounds of PCR. Briefly, the first 389 bp of the RA ATCC11845 dprA gene was amplified from genomic DNA using the primer dprA Comp P1, which contains a Nco I restriction enzyme site, and the primer dprA* P2, which contains mutant nucleotides (TTC). The second 758-bp fragment 
of the $d p r A$ gene was amplified from genomic DNA using the primer dprA* $\mathrm{P} 1$, which contains mutant nucleotides (GAA), and the primer dprA Comp P2, which contains an XhoI restriction enzyme site. The two fragments were then ligated by overlap PCR (Xiong et al., 2006). The resulting amplicon was digested by Nco I/XhoI and cloned into pLMF03 (also digested with NcoI and Xhol) to generate the $\mathrm{pLMF03::} \operatorname{dpr} A^{\mathrm{R} 123 \mathrm{E}}$ plasmid. The ligation products were introduced into $E$. coli strain $\mathrm{DH} 5 \alpha$ cells using the calcium chloride method, and transformants were selected on LB plates containing Amp (100 $\mu \mathrm{g} / \mathrm{ml}$ final concentration). The presence of the correct inserts was confirmed by PCR and sequencing (BGI, Guangzhou, China).

\section{Construction of RA ATCC11845 $\Delta$ dprA::Erm Containing pLMF03::dprA, pLMF03::dprA-SAM-RF, pLMF03::dprA-RF-Z $\alpha$, pLMF03::Sp-dprA, pLMF03::Hp-dprA, pLMF03::Fn-dprA, or pLMF03::dprA ${ }^{\text {R123E }}$ Plasmids}

Because we failed to introduce the plasmid pLMF03 into RA ATCC11845 $\Delta d p r A:: E r m$ using either natural transformation or conjugation, the plasmid was introduced into RA ATCC11845 before $d p r A$ was knocked out. Briefly, the pLMF03::dprA, pLMF03::dprA-SAM-RF, pLMF03::dprA-RF$\mathrm{Z} \alpha$, pLMF03::Sp-dprA, pLMF03::Hp-dprA, pLMF03::Fn-dprA, and $p L M F 03:: d p r A^{\mathrm{R} 123 \mathrm{E}}$ plasmids was introduced into RA ATCC11845, respectively, as described previously (Huang et al., 2017). The transconjugants were selected on blood plates supplemented with Cfx $(1 \mu \mathrm{g} / \mathrm{ml})$. The RA ATCC11845 (pLMF03:: $d p r A)$, RA ATCC11845 (pLMF03::dprA-SAM-RF), RA ATCC11845 (pLMF03::dprA-RF-Z $\alpha)$, RA ATCC11845 (pLMF03::Sp-dprA), RA ATCC11845 (pLMF03::Hp-dprA), RA ATCC11845 (pLMF03::Fn-dprA), and RA ATCC11845 (pLMF03:: $\operatorname{ppr} A^{\mathrm{R} 123 \mathrm{E}}$ ) strains were identified by PCR analysis. Subsequently, the $d \operatorname{pr} A$ gene was deleted as described above (Liu et al., 2017). The transformants were selected on blood plates supplemented with Cfx $(1 \mu \mathrm{g} / \mathrm{ml})$ and Erm $(1 \mu \mathrm{g} / \mathrm{ml})$. The strains, which were designed RA ATCC11845 $\Delta d p r A:: E r m$ (pLMF03::dprA), RA ATCC11845 $\Delta d p r A:: E r m$ (pLMF03::dprASAM-RF), RA ATCC11845 $\Delta d p r A:: E r m$ (pLMF03::dprA-RF-Z $\alpha$ ), RA ATCC11845 $\Delta d p r A:: E r m$ (pLMF03::Sp- $d p r A)$, RA ATCC11 845 $\Delta d p r A:: E r m$ (pLMF03::Hp-dprA), RA ATCC11845 $\Delta d p r A::$ Erm (pLMF03::Fn-dprA), and RA ATCC11845 $\Delta d p r A:: E r m$ (pLM F03::dpr $\left.A^{\mathrm{R} 123 \mathrm{E}}\right)$, respectively, were identified by PCR analysis.

\section{Construction of the Recombinant Vector for Expression}

The complete $d p r A$ and $d p r A^{\text {R123E }}$ genes were amplified by PCR from RA ATCC11845 chromosomal DNA and the recombination vector pLMF03::dprA ${ }^{\mathrm{R} 123 \mathrm{E}}$ using the primers dprAExP1 and dprAExP2his (Table 2). The resulting PCR product was purified, digested with NdeI and XhoI, and ligated into the pET30a plasmid (also digested with $\mathrm{NdeI}$ and XhoI) to generate pET30a::dprA-s and pET30a::dpr $A^{\mathrm{R} 123 \mathrm{E}}$-s plasmids. The ligation products were introduced into $E$. coli strain $\mathrm{DH} 5 \alpha$ cells using the calcium chloride method, and transformants were selected on LB plates containing Kan $(50 \mu \mathrm{g} / \mathrm{ml}$ final concentration). The presence of the correct inserts was confirmed by PCR and sequencing (BGI, Guangzhou, China).

\section{Expression and Purification of DprA and Dpr ${ }^{\text {R123E }}$ His-Tagged Proteins}

The E. coli Rosetta (pET30a::dprA-s) and E. coli Rosetta (pET30a::dpr $A^{\mathrm{R} 123 \mathrm{E}}-\mathrm{s}$ ) strains were grown overnight in LB medium containing Kan $(50 \mu \mathrm{g} / \mathrm{ml})$. Stationary-phase cultures were diluted to an OD600 of 0.05 in $500 \mathrm{ml}$ of LB medium containing Kan $(50 \mu \mathrm{g} / \mathrm{ml})$ and then incubated with shaking at $37^{\circ} \mathrm{C}$ until the culture density reached an OD600 of 0.6. The cells were then induced with $0.05 \mathrm{mM}$ isopropyl b-D-1thiogalactopyranoside (IPTG) and reincubated at $25^{\circ} \mathrm{C}$. The cells were harvested by centrifugation for $10 \mathrm{~min}$ at $8,000 \mathrm{rpm}$ at $4^{\circ} \mathrm{C}$, and the resulting pellet was resuspended in $50 \mathrm{ml}$ of binding buffer I (50 mM Tris- $\mathrm{HCl}, 250 \mathrm{mM} \mathrm{NaCl}, 0.05 \%$ Triton, $\mathrm{pH}$ $8.0)$ containing lysozyme (1 $\mathrm{mg} / \mathrm{ml}$ final concentration) and DNase I (1 U/ml final concentration). The bacteria were lysed by freezing and thawing the cells at least three times. The suspension was then centrifuged at $12,000 \mathrm{rpm}$ for $30 \mathrm{~min}$ at $4^{\circ} \mathrm{C}$, and the supernatant was collected. The supernatant was mixed with $250 \mu \mathrm{l}$ of Ni-NTA-agarose beads according to the manufacturer's instructions. Purified protein was dialyzed twice against a buffer containing $50 \mathrm{mM}$ Tris- $\mathrm{HCl}$ to eliminate any residual imidazole. The protein was stable for at least 1 month when kept at $-80^{\circ} \mathrm{C}$ in $20 \%$ glycerol. Protein concentrations were determined using the BCA method with bovine serum albumin as the standard.

\section{Electrophoretic Mobility Shift Assays (EMSA)}

The DNA-binding activity of DprA and DprA ${ }^{\text {R123E }}$ was measured using an EMSA Kit (Beyotime, China). RA-ssDNA from $_{1}$ RA ATCC11845 (coding sequence: TAGGCTCTGCTAAGGAA GCGTGGGGTCTGTCTAAGTTGGA), RA-ssDNA 2 from RA ATCC11845 (non-coding sequence: AAAAACTACGGAACTG ACTAAAGGCAGAAAAACTAAA CGG), E. coli (EC-ssDNA from $E$. coli (CTCAGGTGCGAAAGCGTGGGGAGCAAA CAGGATTAGATAC) and dsDNA annealed from RA-ssDNA were labeled with biotin using an EMSA Probe Biotin Labeling Kit (Beyotime, China). A $10 \mu \mathrm{l}$ reaction mixture containing $0.5 \mu \mathrm{l}$ of DNA substrate (biotin-labeled ssDNA or dsDNA) in binding buffer with $25 \mathrm{mM}$ HEPES ( $\mathrm{pH} 7.0$ ), $150 \mathrm{mM} \mathrm{NaCl}$, $10 \%$ glycerol, $1 \mathrm{mM}$ DTT and $0.05 \%$ IGEPAL (v/v; SigmaAldrich) mixed with the indicated concentrations of DprA and $\mathrm{DprA}^{\mathrm{R} 123 \mathrm{E}}$. After $30 \mathrm{~min}$ of incubation at $30^{\circ} \mathrm{C}$, the samples were electrophoresed on an $8 \%$ non-denaturing PAGE in $0.5 \times$ TBE (45 mM Tris-borate, $1 \mathrm{mM}$ EDTA, $\mathrm{pH}$ 8.3). A constant voltage of $10 \mathrm{~V} / \mathrm{cm}$ was applied for $2 \mathrm{~h}$ in baths of ice. The gel was transferred to a nylon membrane (Beyotime, China) at $380 \mathrm{~mA}$ for $30 \mathrm{~min}$ in $0.5 \times \mathrm{TBE}$ buffer. Fluorescence detection was performed with streptavidin-conjugated HRP and BeyoECL using a Chemiluminescent EMSA Kit (Beyotime, China) as described in the product specifications. 


\section{qRT-PCR}

Riemerella anatipestifer ATCC11845 (pLMF03), RA ATCC $11845 \Delta d p r A::$ Erm (pLMF03::dprA), and RA ATCC11845 $\Delta d p r A:: E r m$ (pLMF03::dprA ${ }^{\mathrm{R} 123 \mathrm{E}}$ ) were grown in GCB medium at an initial $\mathrm{OD} 600=0.05$ at $37^{\circ} \mathrm{C}$ with shaking at $180 \mathrm{rpm}$. The cells were harvested after $6 \mathrm{~h}$ of incubation. Total RNA was extracted using an RNAprep pure Cell/Bacteria Kit (TIANGEN, China). cDNA was synthesized from each RNA sample as described in a previous study (Liu et al., 2016). Real-time PCR assays were conducted with the primers shown in Table 2. Quantitative PCR was performed in triplicate on deposited samples as described in a previous study (Liu et al., 2016). The RNA quantity was normalized using a probe specific for $16 \mathrm{~S}$ rRNA. Fold change was calculated as described in a previous study with the delta delta Ct method considering the efficiency of the PCR reaction for each target (Pfaffl, 2001).

\section{Statistics and Software}

The results of the transformation experiments were analyzed using GraphPad Prism 7.0 software for Windows (GraphPad Software Inc., La Jolla, CA, United States). The homology analysis of the RF domain of DprA was based on amino acid identity and performed using ClustalX 2.0 or DNAMAN 8.0 (Lynnon Biosoft, ON, Canada). One-way ANOVA followed by Tukey's multiplecomparison test were used to evaluate statistical significance. A $P$-value $<0.05$ was considered significant.

\section{RESULTS}

\section{Identification and Sequence Analysis of DprA in $R$. anatipestifer ATCC11845}

In the genome of RA ATCC11845, RAOC_1073 was annotated as a putative DprA. A protein sequence comparison showed that the DprA of $R$. anatipestifer $\left(\mathrm{DprA}_{\mathrm{Ra}}\right.$ ) had low identity with other well-characterized DprA sequences, including 30\% identity with the DprA of Neisseria meningitidis $\left(\mathrm{DprA}_{\mathrm{Nm}}\right)$, $31 \%$ identity with the DprA of $H$. pylori $\left(\operatorname{DprA}_{\mathrm{Hp}}\right), 37 \%$ identity with the DprA of Streptococcus pneumoniae (DprA $\mathrm{Ap}_{\mathrm{p}}$ ) and $36 \%$ identity with the DprA of Haemophilus influenzae $\left(\mathrm{DprA}_{\mathrm{Hi}}\right)$ The dprA gene (RA0C_1073) is the last gene in a putative operon containing RA0C_1074, RA0C_1075, RA0C_1076, and RA0C_1077 in RA ATCC11845 (Figure 1A), which encode group III truncated hemoglobin, the crossover junction endodeoxyribonuclease RuvC, a hypothetical protein and an Lrp/AsnC family transcriptional regulator, respectively.

Similar to the $\mathrm{DprA}_{\mathrm{Nm}}$ (Hovland et al., 2017) and $\mathrm{DprA}_{\mathrm{Hi}}$ (Karudapuram et al., 1995; Karudapuram and Barcak, 1997), DprARa $_{\mathrm{Ra}}$ has three domains: a sterile alpha motif (SAM), a Rossmann fold (RF) and a winged-helix DNA-binding motif/ Z-DNA-binding domain $(\mathrm{Z} \alpha)$ (Figure 1B). In contrast, $\mathrm{DprA}_{\mathrm{Sp}}$ has only SAM and RF domains and lacks a $Z \alpha$ domain, and $\mathrm{DprA}_{\mathrm{Hp}}$ has only RF and $\mathrm{Z} \alpha$ domains and lacks a SAM domain (Figure 1B). Moreover, among the SAM domains of $\mathrm{DprA}_{\mathrm{Ra}}$, $\mathrm{DprA}_{\mathrm{Nm}}, \mathrm{DprA}_{\mathrm{Sp}}$, and $\mathrm{DprA}_{\mathrm{Hi}}$, the identity ranges from 16 to $17 \%$; while among the $Z \alpha$ domains of $\operatorname{DprA}_{\mathrm{Ra}}, \operatorname{DprA}_{\mathrm{Nm}}, \mathrm{DprA}_{\mathrm{Hp}}$, and $\mathrm{DprA}_{\mathrm{Hi}}$, the identity ranges from 14 to $20 \%$. However, for the RF domain of DprA $A_{R a}, D_{p r A} A_{N m}, D p r A_{s p}, D_{p r A}$, and $D_{p r A} A_{H i}$, the identity ranges from 31 to $37 \%$ (Figure 1C).

\section{DprA Is Essential for the Natural Transformation of $\boldsymbol{R}$. anatipestifer ATCC11845}

In $H$. influenzae, $d p r A$ is necessary for the uptake of chromosomal but not plasmid DNA (Karudapuram et al., 1995). In contrast, deletion of $d p r A$ in $H$. pylori had an impact on transformation of both chromosomal DNA and plasmid DNA (Ando et al., 1999). To test the role of $d p r A$ in the natural transformation of $R$. anatipestifer ATCC11845, the mutant strain RA ATCC11845 $\Delta d p r A::$ Erm and a complementation strain were constructed. Transforming genomic DNA containing resistance to streptomycin as a substrate was prepared. The result showed that the transformation frequency of the wild type was 6 $( \pm 0.7) \times 10^{-5}$. However, no transformation was detected (limitof-detection $\left.=4.2( \pm 1.5) \times 10^{-10}\right)$ in the dprA mutant, and the transformation frequency of the complemented strain was 6.3 $( \pm 1.9) \times 10^{-5}$ (Table 3). These results suggested that the $d p r A$ gene of RA ATCC11845 is essential for natural transformation when genomic DNA was used as the donor. To examine whether the dprA of RA ATCC11845 was also involved in the uptake of plasmids, the plasmid pLMF03, which carries a cefoxitin resistance $\left(\mathrm{Cfx}^{\mathrm{R}}\right)$ marker (Liu et al., 2016), was used as a donor DNA to perform natural transformation. As shown in Table 3, the transformation frequency of the wild-type R. anatipestifer ATCC11845 strain to Cfx ${ }^{\mathrm{R}}$ was $1.7( \pm 0.2) \times 10^{-7}$. Again, no transformation was detected (limit-of-detection $=4.2$ $\left.( \pm 1.5) \times 10^{-10}\right)$ in the $d p r A$ mutant. These results suggest that the dprA of RA ATCC11845 is essential for the transformation of both genomic DNA and plasmid DNA.

\section{The Importance of Three Domains of DprA in Natural Transformation}

As described above, $\mathrm{DprA}_{\mathrm{Ra}}$ has three domains, similar to N. meningitidis and H. influenzae, whereas the DprA of H. pylori and $S$. pneumoniae have only two domains. To investigate the role of each DprA domain in natural transformation, the strain RA ATCC11845 $\Delta d p r A:: E r m$ was complemented by a plasmid expressing SAM-RF domains or a plasmid expressing RF-Z $\alpha$ domains, and the natural transformation frequency was measured. As shown in Figure 2A, the transformation frequency of RA ATCC11845 $\Delta d p r A:: E r m$ (pLMF03::dprA-SAM-RF) was $4.1( \pm 0.7) \times 10^{-6}$, while that of RA ATCC11845 $\Delta$ dprA::Erm (pLMF03::dprA-RF-Z $\alpha)$ was $2.4( \pm 0.5) \times 10^{-8}$. Both partially restored the level of natural transformation in the mutant strain RA ATCC11845 $\Delta d p r A:: E r m$. Interestingly, the transformation frequency of RA ATCC11845 $\Delta d p r A::$ Erm (pLMF03::dprA-SAMRF) was higher than that of RA ATCC11845 $\Delta d p r A:: E r m$ (pLMF03::dprA-RF-Z $\alpha$ ). From these results, we predicted that the role of the SAM domain is more important role than that of the $\mathrm{Z} \alpha$ domain in the natural transformation of RA ATCC11845.

To further verify the functions of these three domains in natural transformation, the strain RA ATCC11845 $\Delta d p r A::$ Erm 


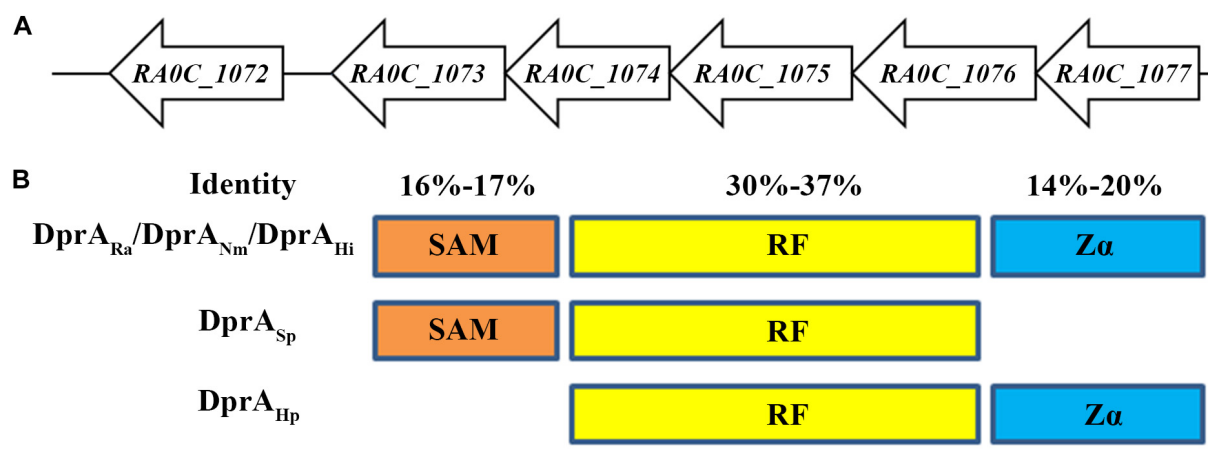

C

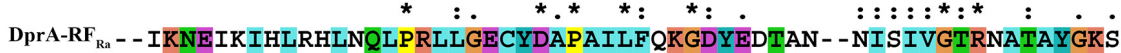
DprA-RF $_{\mathrm{s}_{\mathrm{p}}}$ DPRA-RF ${ }_{\mathrm{HP}}$ MNQRMKSHFQYSTLENIPKAFDILKDPPKKLYCVGDTKLLDTPLKVAI IGTRRPTPY SKQ

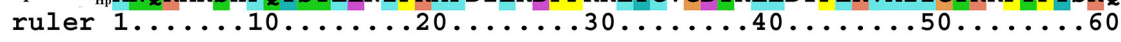

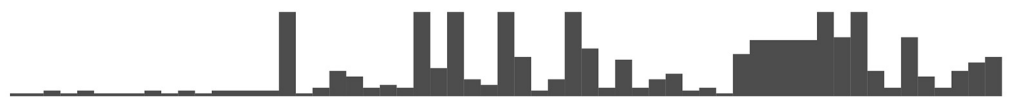

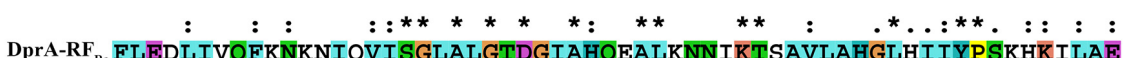
DprA-RF $\mathrm{F}_{\mathrm{p}}$ SVEKVIQGLEN - ELVIVSGLAKGIDTAAHMAALQNGGKTIAVIGTGLDVFYPKANKRLQD

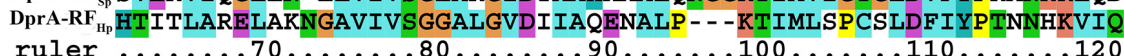

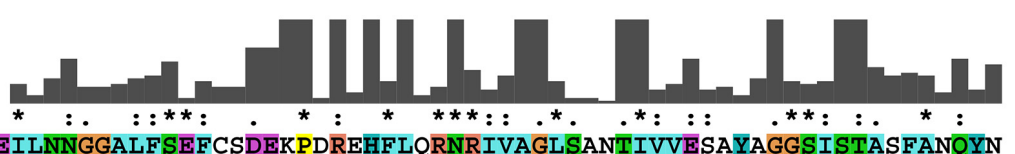
DPRA-RF ${ }_{\mathrm{Ra}}$ EILNNGGALFSEFCSDEKPDREHFLQRNRIVAGLSANTIVVESAYAGGSISTASFANQYN DprA-RF $\mathrm{s}_{\mathrm{p}} \mathrm{YIG-NDHLVLSEYGPGEQPLKFHF} \mathrm{PARNRI} \mathrm{IAGLCRGVIVAEAKMRSGSLITCERAMEEG}$ DprA-RF ${ }_{\text {нр }}$ EIA-QNGLILSEYEKDFMPI KGSFLARNRLVIALSDVVI I PQADLKSGSMSSARLAQKYQ ruler $\ldots \ldots 130 \ldots \ldots 140 \ldots \ldots 150 \ldots \ldots 160 \ldots \ldots 170 \ldots \ldots 180$

: : : : : : : * : $:$ * $^{*}:$. $^{*}: \quad$ : $: \quad:^{*}:$. :

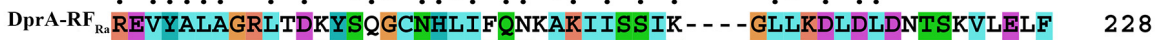

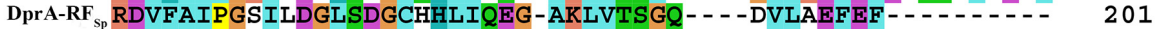
DPrA-RF $_{\text {Hp }}$ KPLFVLPQRLNE - - SDGTNELLEKGQAQGIFNIQNF INTLLKDYHLKEMPEMEDEF 230

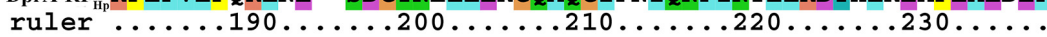

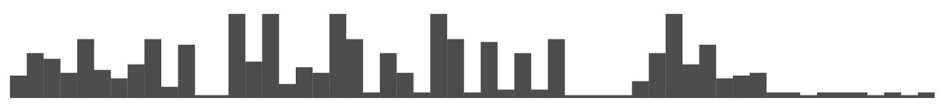

FIGURE 1 | Bioinformatics analysis of DprA $\mathrm{Ra}$. (A) The dprA locus in R. anatipestifer ATCC11845. ORFs are indicated by block arrows and point in the direction of transcription. Names of ORFs are indicated below each arrow. (B) Predicted overall domain structure of DprARa and orthologs of $S$. pneumonia (DprA $\mathrm{Sp}$ ), $H$. pylori $\left(D_{p r} A_{H p}\right)$, and Neisseria meningitidis (DprA $\mathrm{Nm}_{\mathrm{m}}$ ). (C) Deduced amino acid sequence alignment of the hallmark RF DprA domains of $R$. anatipestifer (RA),

S. pneumoniae (Sp), and H. pylori (Hp); alignment was performed using ClustalX 2.0 *the same amino acid.

was complemented by either a $d p r A$ of $S$. pneumoniae containing SAM and RF domains or a $\operatorname{dpr} A$ of $H$. pylori containing RF and $\mathrm{Z} \alpha$ domains, and the natural transformation frequency was measured. As shown in Figure 2B, the transformation frequency of RA ATCC11845 $\Delta d p r A:: E r m$ (pLMF03::Sp-dprA) was $3.8( \pm 0.9) \times 10^{-7}$, while that of RA ATCC11845 $\Delta d p r A:: E r m$ $($ pLMF03::Hp- $d p r A)$ was $2.9( \pm 0.6) \times 10^{-8}$. Interestingly, the frequency of complementing in cells the $d p r A$ from S. pneumoniae was higher than that observed in cells with the dprA from $H$. pylori. These results suggest that the role of the SAM domain is more important than that of the $\mathrm{Z} \alpha$ domain in natural transformation.

\section{The Amino Acid Arg123 in the RF Domain Is Essential for the Function of DprA in RA ATCC11845}

As described above, it has been hypothesized that the RF domain is essential for the function of DprA in natural transformation. Moreover, sequence comparison showed that the amino acid residue $\operatorname{Arg} 123$ is one of the most conserved amino acid residues in this domain (Figure 1C). Thus, we chose to explore the function of Arg123 in natural transformation in RA ATCC11845. A mutant $d p r A^{\mathrm{R} 123 \mathrm{E}}$ (arginine mutated to glutamic acid) construct was developed and cloned into the shuttle plasmid 
TABLE 3 | Natural transformation assays performed in RA ATCC11845, RA ATCC11845 $\Delta d p r A:: E r m$, RA ATCC11845 $d$ prA::Erm (pLMF03::dprA), and RA ATCC11845 $\Delta d p r A:: E r m$ (pLMF03::dprA $\left.{ }^{\mathrm{R} 123 \mathrm{E}}\right)$.

\begin{tabular}{|c|c|c|}
\hline Strain & $\begin{array}{c}\text { Transformation } \\
\text { frequency using } \\
\text { chromosomal DNA }\end{array}$ & $\begin{array}{c}\text { Transformation } \\
\text { frequency using } \\
\text { plasmid DNA }\end{array}$ \\
\hline RA ATCC11845 & $6( \pm 0.7) \times 10^{-5}$ & $1.7( \pm 0.2) \times 10^{-7}$ \\
\hline RA ATCC11845 $\Delta d p r A:: E r m$ & $<$ d.l. & $<$ d.l. \\
\hline $\begin{array}{l}\text { RA ATCC11845 } \Delta d p r A:: E r m \\
\text { (pLMF03::dprA) }\end{array}$ & $6.3( \pm 1.9) \times 10^{-5}$ & NA \\
\hline $\begin{array}{l}\text { RA ATCC11845 } \Delta d p r A:: \text { Erm } \\
\text { (pLMF03::dprA }{ }^{\mathrm{R} 123 \mathrm{E}} \text { ) }\end{array}$ & $<$ d.l. & NA \\
\hline
\end{tabular}

NA, Not applicable. <d.I., below detection limit. The average detection limit of nontransformable strains was $4.2( \pm 1.5) \times 10^{-10}$.

pLMF03 as described in the Section "Materials and Methods". As shown in Table 3, the plasmid pLMF03::dprA ${ }^{\mathrm{R} 123 \mathrm{E}}$ was not able to restore natural transformation to RA ATCC11845 $\Delta d p r A:: E r m$. As a control, $d p r A^{\mathrm{R} 123 \mathrm{E}}$ was transcribed well in the RA ATCC11845 $\Delta d p r A:: E r m$ strain (pLMF03::dprA ${ }^{\mathrm{R} 123 \mathrm{E}}$; Supplementary Figure $\mathrm{S} 1$ ). The results suggested that the RF domain is essential for natural transformation in RA ATCC11845 and that the amino acid Arg123 plays a key role in this domain.

\section{DprA ${ }^{\text {R123E }}$ Has a Dramatic Effect on Binding ssDNA and dsDNA}

The R123E mutant of DprA abolished the ability of RA ATCC11845 to undergo natural transformation, potentially because the mutant was unable to bind and protect DNA. Thus, we next evaluated whether Arg123 of the DprA domain of RA
ATCC11845 affects the ability of the protein to bind to DNA. In these experiments, DprA and DprA ${ }^{\mathrm{R} 123 \mathrm{E}}$ were expressed and purified, and EMSA was performed as described in the Section "Materials and Methods". In this study, we first chose three different sources of single strand DNA (ssDNA): RA-ssDNA from the coding sequence of RA ATCC11845, RA-ssDNA 2 from the non-coding sequence of RA ATCC11845 and (EC-ssDNA from $E$. coli. The sequences of those ssDNA were shown in the Section "Materials and Methods." Single-strand DNA (ssDNA) was synthesized and used as a substrate for EMSA. As shown in Figures 3A-C, all the ssDNA was shifted in the presence of DprA. As the concentration of DprA increased, the formation of protein-DNA complexes was clearly slowed, and this change was associated with a parallel loss of uncomplexed DprA and ssDNA, suggesting that DprA can bind ssDNA and that it has no sequence or species specificity.

Interestingly, DprA of $H$. pylori binds not only ssDNA but also dsDNA (Dwivedi et al., 2013). However, DprA of S. pneumoniae and Bacillus subtilis has been reported to bind and protect ssDNA but not dsDNA (Mortier-Barriere et al., 2007). To determine whether DprA of $R$. anatipestifer binds dsDNA, EMSA was performed using both DprA and biotinylated dsDNA. The dsDNA (40 bp) was annealed from RA-ssDNA $A_{1}$. As shown in Figure 4A, the retarded protein-DNA complex became more evident with the increasing of DprA concentration, suggesting that DprA binds dsDNA. As shown in Figures 3D-F, 4B, there is not any retarded protein-DNA complex as the concentration of the $\mathrm{DprA}^{\mathrm{R} 123 \mathrm{E}}$ was increased as the concentration of $\mathrm{DprA}^{\mathrm{R} 123 \mathrm{E}}$ increased. Taken together, these results suggest that the Arg123 mutant abolished the ability of DprA to bind and protect DNA.

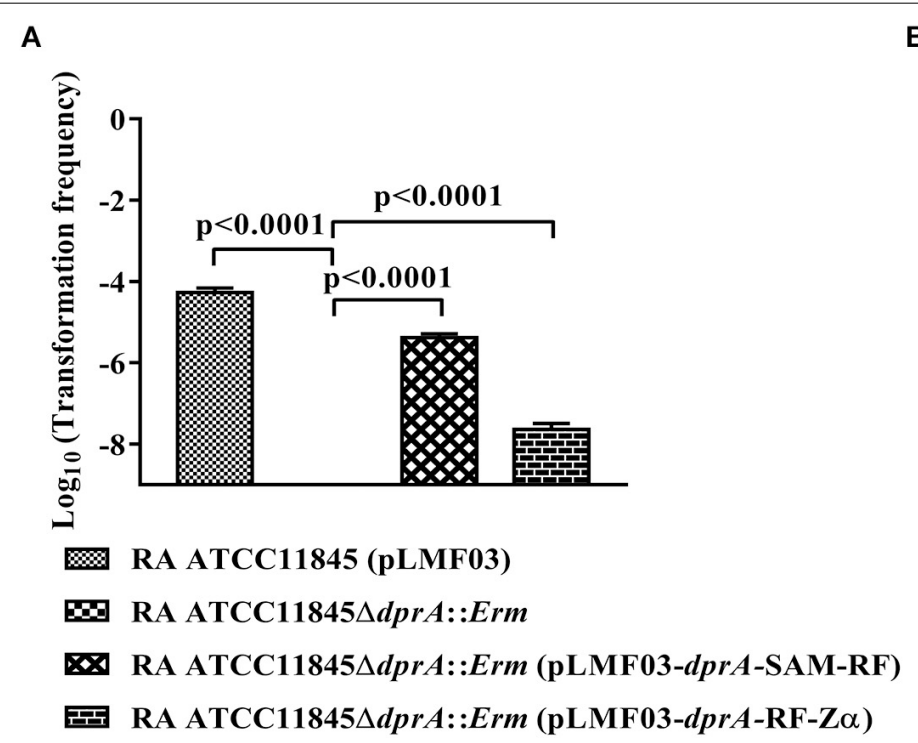

B

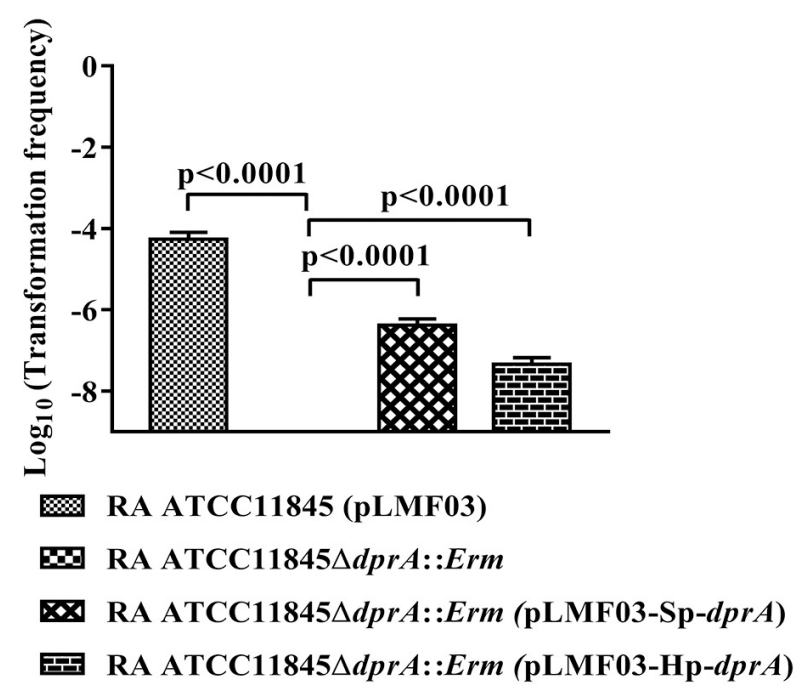

FIGURE 2 | Complementation of $R$. anatipestifer ATCC11845 $\Delta d p r A:$ :Erm by different domains of DprARa, dprA of S. pneumoniae and dprA of $H$. pylori. (A) Complementation of $R$. anatipestifer ATCC11845 $\Delta d p r A:: E r m$ by different domains of DprARa. (B) Complementation of R. anatipestifer ATCC11845 $\Delta d p r A::$ Erm by dprA of S. pneumoniae (Sp-dprA) and dprA of H. pylori ( $\mathrm{Hp}-d p r A)$. The $\log _{10}$ of averages and standard deviations of three independent experiments are shown. The numbers above each data point represent $P$-values for comparisons (one-way ANOVA followed by Tukey's multiple-comparison test) of log ${ }_{10}$ of the average relative transformation frequencies. 
A

$\begin{array}{lllllll}0 & 1 & 5 & 10 & 15 & 20 & \mu \mathrm{M}\end{array}$

B

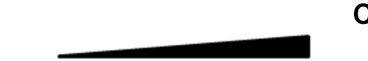

C

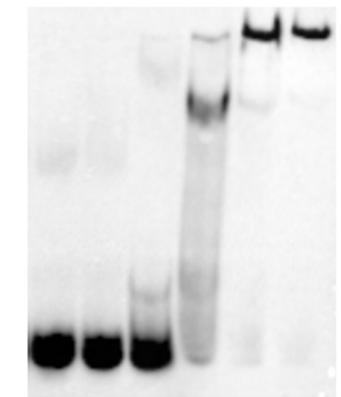

D

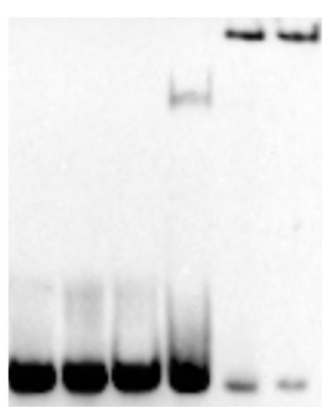

E

$\begin{array}{lllllll}0 & 1 & 5 & 10 & 15 & 20 & \mu \mathrm{M}\end{array}$

$\begin{array}{llllll}0 & 1 & 5 & 10 & 15 & 20\end{array}$

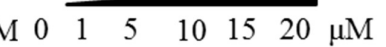

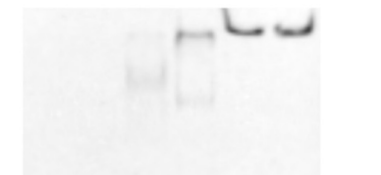

అே0 -

$\mathbf{F}$

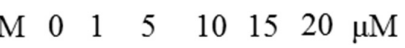

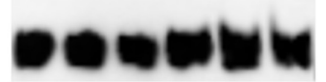
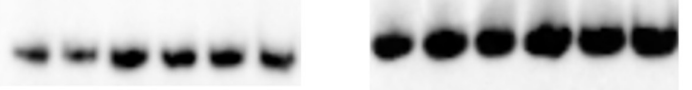

FIGURE 3 | The effect of the R123E mutant of DprA on binding to single-strand DNA (SSDNA). All SSDNA was labeled by biotin. The first lane of each picture contains free ssDNA without proteins. Different concentrations of DprA (1-20 $\mu \mathrm{M})$ were combined with $0.5 \mu$ l of DNA substrate (biotin-labeled ssDNA) and incubated for $30 \mathrm{~min}$ at $30^{\circ} \mathrm{C}$ before the mixtures were loaded onto the gel. (A) Interaction between DprA and ssDNA from the $R$. anatipestifer coding sequence (RA-ssDNA $A_{1}$ ). (B) Interaction between DprA and ssDNA from the R. anatipestifer non-coding sequence (RA-ssDNA $)$ ). (C) Interaction between DprA and ssDNA from E. coli XL1-blue (E. coli-ssDNA). (D) Interaction between DprA ${ }^{\mathrm{R} 123 \mathrm{E}}$ and ssDNA from the $R$. anatipestifer coding sequence (RA-ssDNA $\mathrm{A}_{1}$ ). (E) Interaction between $\operatorname{DprA}^{\mathrm{R} 123 \mathrm{E}}$ and ssDNA from the $R$. anatipestifer non-coding sequence $\left(\mathrm{RA}-\mathrm{ssDNA}\right.$ ). (F) Interaction between DprA ${ }^{\mathrm{R} 123 \mathrm{E}}$ and ssDNA from E. coli XL1-blue (E. coli-ssDNA). Samples were electrophoresed on an $8 \%$ non-denaturing PAGE gel and detected by fluorography.

A

0
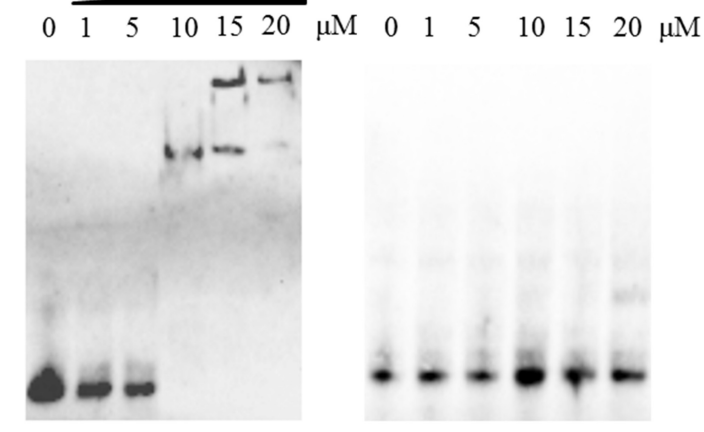

FIGURE 4 | The effect of the R123E mutant of DprA on binding to double-strand DNA (dsDNA). The dsDNA was annealed from RA-ssDNA 1 and labeled with biotin. The first lane of each picture contained free dsDNA without proteins. Different concentrations of DprA $(1-20 \mu \mathrm{M})$ were combined with $0.5 \mu$ l of DNA substrate (biotin-labeled dsDNA) and incubated for 30 min at $30^{\circ} \mathrm{C}$ before the mixture was loaded on the gel. (A) Interaction between DprA and dsDNA. (B) Interaction between DprA ${ }^{\text {R123E }}$ and dsDNA. Samples were electrophoresed on an 8\% non-denaturing PAGE gel and detected by fluorography.

\section{DprA Has a Conserved Function in Evolution}

DprA has been found in nearly all the sequenced bacteria, including bacteria currently recognized as non-competent (Supplementary Table S1). The identity ranged from 35 to 63\% in Flavobacteriaceae (Supplementary Table S1). Compared to other bacterial genera, for RA ATCC11845, the identity ranged from 28 to 39\%, but it shared 59\% identity with Spirochaetes. As shown in Figure 5, a phylogenetic analysis of DprA across different bacterial species showed that there were no obvious branches between naturally competent bacteria and non-competent bacteria. It has been speculated that DprA has conserved functions across different bacteria. Thus, we wondered whether $d p r A$ from other bacteria would be able to restore natural transformation to RA ATCC11845 $\Delta d p r A:: E r m$. In a proof-ofprinciple experiment, we chose the DprA of $F$. naviforme, which has not been reported natural competence currently and has low identity (30\%) with the DprA of RA ATCC11845 (Supplementary Figure S3). The dprA of F. naviforme was synthesized and cloned into the shuttle plasmid pLMF03. An RA ATCC11845 $\triangle d p r A::$ Erm construct that expressed 


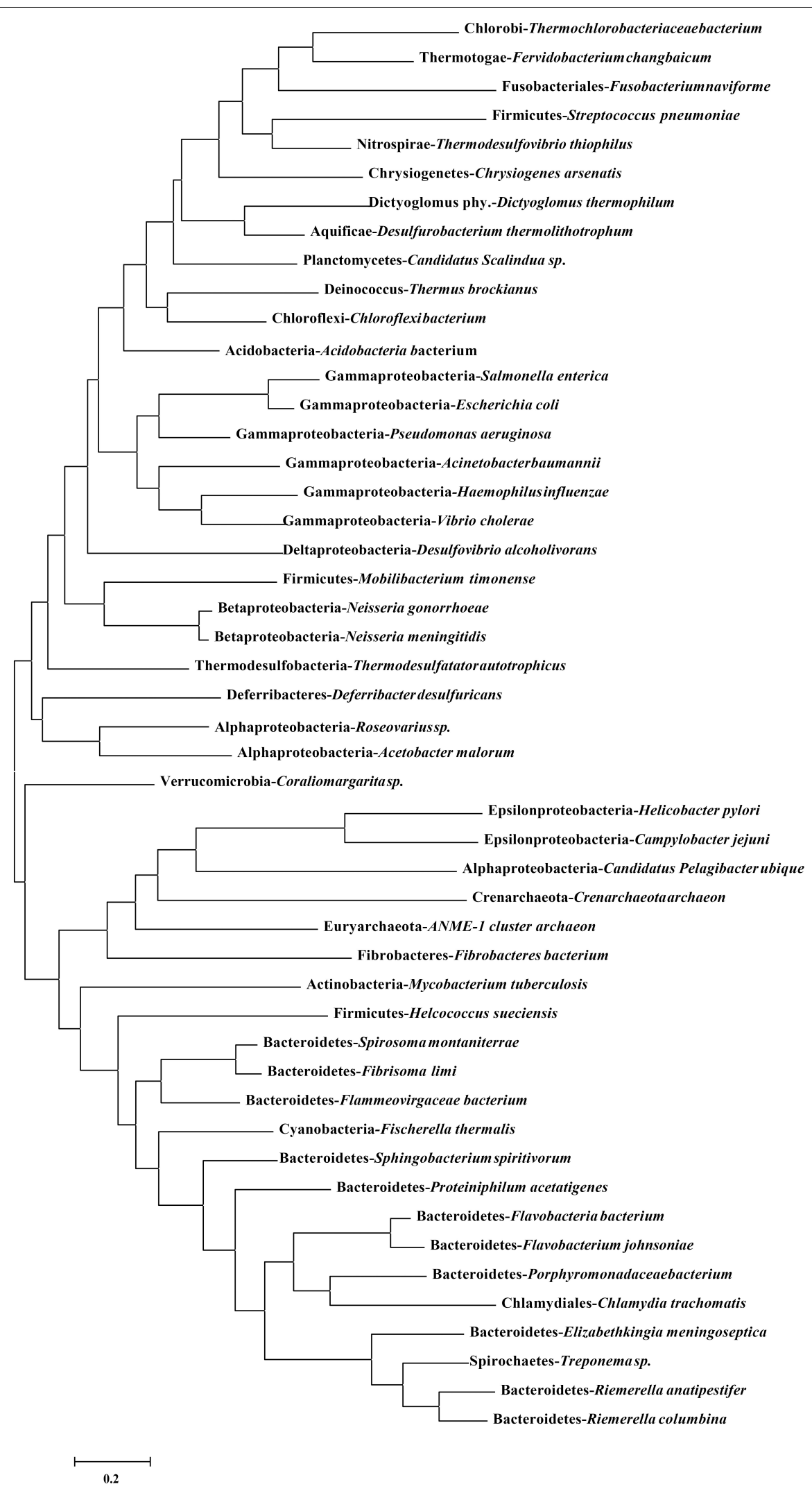

FIGURE 5 | Phylogenetic analysis of DprA among different bacterial species. A phylogenetic tree was constructed based on amino acid sequences using MEGA6.0 using neighbor-joining method. The sequence information for DprA is listed in Supplementary Table S1. 


\section{है \\ RA ATCC11845 (pLMF03) \\ RA ATCC11845 $\triangle$ dprA::Erm \\ 区 RA ATCC11845 $\triangle d p r A:: E r m$ (pLMF03-Fn-dprA)}

FIGURE 6 | Complementation of $R$. anatipestifer ATCC11845 $\Delta d p r A:: E r m$ by the dprA of the non-competent bacterium F. naviforme. The $\log _{10}$ of averages and standard deviations of three independent experiments are shown. The numbers above each data point represent $P$-values for comparisons (one-way ANOVA followed by Tukey's multiple-comparison test) of $\log _{10}$ of the average relative transformation frequencies.

pLMF03::Fn-dprA was constructed as described in the Section "Materials and Methods". As shown in Figure 6, the transformation frequency of RA ATCC11845 (pLMF03) was $2.9( \pm 0.3) \times 10^{-5}$, while that of RA ATCC11845 $\Delta$ dprA::Erm $($ pLMF03::Fn- $d p r A)$ was $4.9( \pm 0.7) \times 10^{-7}$. These results suggest that a $d p r A$ from $F$. naviforme, partially restored natural transformation to the mutant RA ATCC11845 $\Delta$ dprA::Erm.

\section{DISCUSSION}

Natural transformation is a widely distributed mechanism used by many bacterial genera to acquire DNA and undergo genetic recombination (Johnston et al., 2014). The competence machinery actively processes exogenous dsDNA and takes up internalized ssDNA to replace homologous (or partially homologous) chromosomal sequences via a mechanism catalyzed by RecA and facilitated by accessory factors, such as DprA (Kidane et al., 2012). Although it has been found that $R$. anatipestifer was natural competent and established multiple genome editing tools using it (Liu et al., 2018b), the mechanism of natural transformation in $R$. anatipestifer kept largely unknown. This study focused on the characterization of DprA in R. anatipestifer ATCC11845.

DprA is essential for the natural transformation in $N$. meningitidis, N. gonorrhoeae, S. pneumoniae, and H. influenzae (Karudapuram et al., 1995; Berge et al., 2003; Duffin and Barber, 2016; Hovland et al., 2017). However, in B. subtilis, $d p r A$ is not stringently required for DNA transformation as there is some redundancy between the $\mathrm{RecF}$ and DprA pathways (Kidane et al., 2009). RA0C_1073 of RA ATCC11845 encodes a hypothetical DprA with low identity to the DprA sequences of other bacteria. To explore whether this gene is involved in natural transformation in RA ATCC11845, a $d p r A$ mutant strain was constructed. The transformation frequency of $d p r A$ mutant strain was not able to be detected whatever plasmid or chromosomal DNA was used as the donor DNA, suggesting that DprA is essential for the natural transformation of both chromosomal DNA and plasmid DNA in RA ATCC11845, consistent with what has been observed in H. pylori (Ando et al., 1999), but inconsistent with $H$. influenzae, in which $d p r A$ is necessary for chromosomal uptake but not for plasmid (Karudapuram et al., 1995). In the case of H. influenzae, it was hypothesized that the plasmid DNA enters the cytoplasm without cutting and recombination. However, in the case of $R$. anatipestifer, we predicted that the plasmid DNA enter the cytoplasm as the single strand DNA and was re-circled, since if the circular DNA enters into the cytoplasm directly, the $d p r A$ mutant should have no any effect on transformation frequency of plasmid. However, the fact is that the plasmid was not able to be introduced into the $d p r A$ mutant by natural transformation after many times attempts. Additionally, we tried many times to introduce the plasmid into $d p r A$ mutant by conjugation. It was also failed. Although it is impossible that natural transformation and conjugation use the same pathway, it is predicted that single-stranded linear of the plasmid is transported into the cytoplasm by conjugation and the $\operatorname{dpr} A$ was required for the recyclizing.

Bioinformatic analysis showed that $\mathrm{DprA}_{\mathrm{Ra}}$ has three domains (SAM, RF and $\mathrm{Z} \alpha$ domains). To investigate the function of each domain of DprA, complementary assays were performed with SAM-RF domain and RF-Z $\alpha$ domain, respectively. It was showed that the natural transformation frequency was higher when the mutant strain was complemented by SAM-RF domain, than that complemented by RF-Z $\alpha$ domain. Consistently, the $\operatorname{dpr} A$ of H. pylori (Hp-dprA), which contains SAM and RF domains, was more efficiently to restore the natural transformation of the $d p r A$ mutant strain than $d p r A$ of $S$. pneumoniae (Sp-dprA), which contains RF and $\mathrm{Z} \alpha$ domain, did. All these results suggest that the SAM domain is more important than $Z \alpha$ domain for natural transformation. It is possible that the SAM domain play more important role than $\mathrm{Z} \alpha$ domain in protect ssDNA or promote recombination. In DprAsp, the SAM domain plays a role in intracellular signaling and the regulation of competence, in which it plays an important role in shutting down natural competence in this bacterium by interacting with ComE (Mirouze et al., 2013). Compared with S. pneumonia, there are some different traits in $R$. anatipestifer. First, the natural competence of $R$. anatipestifer is constitutive. Second, the SAM domain in $D_{\text {prA }}$ Ra lacks the amino acid residues that confer the ability to induce competence in S. pneumoniae (Supplementary Figure S2). Third, $R$. anatipestifer lacks the ComDE twocomponent system, which is responsible for regulating natural competence in S. pneumoniae (Martin et al., 2013). The specific function that the SAM domain of RA ATCC11845 plays in natural transformation is unknown at this time. It has been hypothesized that the SAM domain of DprA in R. anatipestifer protects ssDNA from degradation or interacts with RecA to promote homologous recombination. 
When the conserved amino acid Arg123 in the RF domain of DprA of RA ATCC11845 was switched to glutamic acid (Glu), the resulting Arg123 mutant completely abolished the ability of RA ATCC11845 to undergo natural transformation, suggesting that RF is essential for natural transformation in $R$. anatipestifer. It has also been shown that Arg123 is essential for the function of DprA $\mathrm{Ra}$. To explore whether the abrogation of natural transformation observed in the Arg123 mutant strains was because the mutant was no longer able to bind DNA, EMSA was performed to evaluate the interaction between ssDNA and DprA or DprA ${ }^{\mathrm{R} 123 \mathrm{E}}$. The results showed that DprA binds ssDNA without sequence or species specificity. Moreover, DprA can also bind dsDNA, consistent with H. pylori (Dwivedi et al., 2013). As expected, DprA ${ }^{\mathrm{R} 123 \mathrm{E}}$ was no longer able to bind either ssDNA or dsDNA. In this study, it was shown that RF was essential for natural transformation and that the Arg123 site in the RF domain was essential for the functions of DprA in R. anatipestifer ATCC11845, which is consistent with findings in $H$. pylori. In H. pylori, the 3D structure of DprA was studied, and the results showed that $\operatorname{Arg} 52$ is essential for $\mathrm{DprA}_{\mathrm{Hp}}$ to grasp the substrate with high affinity (Wang W. et al., 2014).

Phylogenetic assay showed that the homolog of DprA was distributed in most of sequenced bacterial species, including the bacteria that was not found to be natural competent at present. It was consistent with the previous study which suggested that natural competence is an ancient ancestral trait and is usually to be lost during the evolution (Redfield et al., 2006). This viewpoint was strengthened by the fact that the $d p r A$ of $F$. naviforme, which has not been reported natural competence currently, was able to partially restored natural transformation to $R$. anatipestifer $d p r A$ mutant. It has been reported that DprA interact with RecA to promote recombination (Mortier-Barriere et al., 2007). There is a possible reason for explaining why $\operatorname{dpr} A$ of $F$. naviforme only partially restore the ability of natural competence of $R$. anatipestifer. Taken together, $R$. anatipestifer is the first bacterium to be identified as a naturally competent species in Flavobacteriaceae. In this study, our experiments involving DprA were helpful for revealing the mechanism of natural

\section{REFERENCES}

Ando, T., Israel, D. A., Kusugami, K., and Blaser, M. J. (1999). HP0333, a member of the dprA family, is involved in natural transformation in Helicobacter pylori. J. Bacteriol. 181, 5572-5580.

Berge, M., Mortier-Barriere, I., Martin, B., and Claverys, J. P. (2003). Transformation of Streptococcus pneumoniae relies on DprA- and RecA-dependent protection of incoming DNA single strands. Mol. Microbiol. 50, 527-536.

Chen, I., and Dubnau, D. (2004). DNA uptake during bacterial transformation. Nat. Rev. Microbiol. 2, 241-249. doi: 10.1038/nrmicro844

Draskovic, I., and Dubnau, D. (2005). Biogenesis of a putative channel protein, ComEC, required for DNA uptake: membrane topology, oligomerization and formation of disulphide bonds. Mol. Microbiol. 55, 881-896. doi: 10.1111/j. 1365-2958.2004.04430.x

Dubnau, D. (1999). DNA uptake in bacteria. Annu. Rev. Microbiol. 53, 217-244. doi: 10.1146/annurev.micro.53.1.217

Duffin, P. M., and Barber, D. A. (2016). DprA is required for natural transformation and affects pilin variation in Neisseria gonorrhoeae. Microbiology 162, 16201628. doi: $10.1099 / \mathrm{mic} .0 .000343$ transformation in $R$. anatipestifer, even in Flavobacteriaceae. These findings will also be helpful for improving gene editing methods for $R$. anatipestifer.

\section{AUTHOR CONTRIBUTIONS}

ML, AC, and FB conceived and designed the experiments. LH, XT, DZ, MW, YL, LZ, XC, and YY performed the experiments. LP, MR, JH, RJ, SC, and XZ analyzed the data. BT, YW, $\mathrm{QY}$, and SZ contributed reagents, materials, and analysis tools. $\mathrm{LH}, \mathrm{ML}, \mathrm{FB}$, and $\mathrm{AC}$ wrote the manuscript. All authors have reviewed the manuscript.

\section{FUNDING}

This work was supported by the National Natural Science Foundation of China (31572521), the China Agricultural Research System (CARS-42-17), the Sichuan Veterinary Medicine and Drug Innovation Group of China Agricultural Research System (CARS-SVDIP), and a Special Fund for Key Laboratory of Animal Disease and Human Health of Sichuan Province (2016JPT0004).

\section{ACKNOWLEDGMENTS}

We thank Qiong Liu (Nanchang University) and Hebin Liao (North Sichuan Medical College) for generously providing the templates for $H$. pylori and S. pneumoniae, respectively.

\section{SUPPLEMENTARY MATERIAL}

The Supplementary Material for this article can be found online at: https://www.frontiersin.org/articles/10.3389/fgene. 2019.00429/full\#supplementary-material

Dwivedi, G. R., Sharma, E., and Rao, D. N. (2013). Helicobacter pylori DprA alleviates restriction barrier for incoming DNA. Nucleic Acids Res. 41, 32743288. doi: 10.1093/nar/gkt024

Hobbs, M., and Mattick, J. S. (1993). Common components in the assembly of type 4 fimbriae, DNA transfer systems, filamentous phage and proteinsecretion apparatus: a general system for the formation of surface-associated protein complexes. Mol. Microbiol. 10, 233-243. doi: 10.1111/j.1365-2958.1993. tb01949.x

Hovland, E., Beyene, G. T., Frye, S. A., Homberset, H., Balasingham, S. V., GomezMunoz, M., et al. (2017). DprA from Neisseria meningitidis: properties and role in natural competence for transformation. Microbiology 163, 1016-1029. doi: 10.1099/mic.0.000489

Huang, L., Yuan, H., Liu, M. F., Zhao, X. X., Wang, M. S., Jia, R. Y., et al. (2017). Type B chloramphenicol acetyltransferases are responsible for chloramphenicol resistance in Riemerella anatipestifer. China Front. Microbiol. 8:297. doi: 10. 3389/fmicb.2017.00297

Johnston, C., Martin, B., Fichant, G., Polard, P., and Claverys, J. P. (2014). Bacterial transformation: distribution, shared mechanisms and divergent control. Nat. Rev. Microbiol. 12, 181-196. doi: 10.1038/nrmicro3199 
Karudapuram, S., and Barcak, G. J. (1997). The Haemophilus influenzae dprABC genes constitute a competence-inducible operon that requires the product of the tfoX (sxy) gene for transcriptional activation. J. Bacteriol. 179, 4815-4820.

Karudapuram, S., Zhao, X., and Barcak, G. J. (1995). DNA sequence and characterization of Haemophilus influenzae dprA+, a gene required for chromosomal but not plasmid DNA transformation. J. Bacteriol. 177, 3235-3240.

Kidane, D., Ayora, S., Sweasy, J. B., Graumann, P. L., and Alonso, J. C. (2012). The cell pole: the site of cross talk between the DNA uptake and genetic recombination machinery. Crit. Rev. Biochem. Mol. Biol. 47, 531-555. doi: 10.3109/10409238.2012.729562

Kidane, D., Carrasco, B., Manfredi, C., Rothmaier, K., Ayora, S., Tadesse, S., et al. (2009). Evidence for different pathways during horizontal gene transfer in competent Bacillus subtilis cells. PLoS Genet. 5:e1000630. doi: 10.1371/journal. pgen. 1000630

Kristensen, B. M., Sinha, S., Boyce, J. D., Bojesen, A. M., Mell, J. C., and Redfield, R. J. (2012). Natural transformation of Gallibacterium anatis. Appl. Environ. Microbiol. 78, 4914-4922. doi: 10.1128/AEM.00412-12

Liu, M., Huang, M., Shui, Y., Biville, F., Zhu, D., Wang, M., et al. (2018a). Roles of B739_1343 in iron acquisition and pathogenesis in Riemerella anatipestifer CH-1 and evaluation of the RA-CH-1DeltaB739_1343 mutant as an attenuated vaccine. PLoS One 13:e0197310. doi: 10.1371/journal.pone.0197310

Liu, M., Huang, Y., Liu, J., Biville, F., Zhu, D., Wang, M., et al. (2018b). Multiple genetic tools for editing the genome of Riemerella anatipestifer using a counterselectable marker. Appl. Microbiol. Biotechnol. 102, 7475-7488. doi: 10.1007/s00253-018-9181-4

Liu, M., Wang, M., Zhu, D., Wang, M., Jia, R., Chen, S., et al. (2016). Investigation of TbfA in Riemerella anatipestifer using plasmid-based methods for gene over-expression and knockdown. Sci. Rep. 6:37159. doi: 10.1038/srep37159

Liu, M., Zhang, L., Huang, L., Biville, F., Zhu, D., Wang, M., et al. (2017). Use of natural transformation to establish an easy knockout method in Riemerella anatipestifer. Appl. Environ. Microbiol. 83:e127-17. doi: 10.1128/AEM.00127-17

Luo, H., Liu, M., Wang, L., Zhou, W., Wang, M., Cheng, A., et al. (2015). Identification of ribosomal RNA methyltransferase gene ermF in Riemerella anatipestifer. Avian Pathol. 44, 162-168. doi: 10.1080/03079457.2015.1019828

Luo, H. Y., Liu, M. F., Wang, M. S., Zhao, X. X., Jia, R. Y., Chen, S., et al. (2018). A novel resistance gene, $\operatorname{lnu}(\mathrm{H})$, conferring resistance to lincosamides in Riemerella anatipestifer CH-2. Int. J. Antimicrob. Agents 51, 136-139. doi: 10.1016/j.ijantimicag.2017.08.022

Martin, B., Soulet, A. L., Mirouze, N., Prudhomme, M., Mortier-Barriere, I., Granadel, C., et al. (2013). ComE/ComE $\sim \mathrm{P}$ interplay dictates activation or extinction status of pneumococcal X-state (competence). Mol. Microbiol. 87, 394-411. doi: $10.1111 / \mathrm{mmi} .12104$

Mell, J. C., and Redfield, R. J. (2014). Natural competence and the evolution of DNA uptake specificity. J. Bacteriol. 196, 1471-1483. doi: 10.1128/JB.01293-13

Miller, V. L., and Mekalanos, J. J. (1988). A novel suicide vector and its use in construction of insertion mutations: osmoregulation of outer membrane proteins and virulence determinants in Vibrio cholerae requires toxR. J. Bacteriol. 170, 2575-2583.

Mirouze, N., Berge, M. A., Soulet, A. L., Mortier-Barriere, I., Quentin, Y., Fichant, G., et al. (2013). Direct involvement of DprA, the transformation-dedicated RecA loader, in the shut-off of pneumococcal competence. Proc. Natl. Acad. Sci. U.S.A. 110, E1035-E1044. doi: 10.1073/pnas.1219868110
Mortier-Barriere, I., Velten, M., Dupaigne, P., Mirouze, N., Pietrement, O., McGovern, S., et al. (2007). A key presynaptic role in transformation for a widespread bacterial protein: DprA conveys incoming ssDNA to RecA. Cell 130, 824-836. doi: 10.1016/j.cell.2007.07.038

Pfaffl, M. W. (2001). A new mathematical model for relative quantification in real-time RT-PCR. Nucleic Acids Res. 29:e45.

Redfield, R. J., Findlay, W. A., Bosse, J., Kroll, J. S., Cameron, A. D., and Nash, J. H. (2006). Evolution of competence and DNA uptake specificity in the Pasteurellaceae. BMC Evol. Biol. 6:82. doi: 10.1186/1471-2148-6-82

Song, X. H., Zhou, W. S., Wang, J. B., Liu, M. F., Wang, M. S., Cheng, A. C., et al. (2016). Genome sequence of Riemerella anatipestifer strain RCAD0122, a multidrug-resistant isolate from ducks. Genome Announc. 4:e00332-16. doi: 10.1128/genomeA.00332-16

Wang, W., Ding, J., Zhang, Y., Hu, Y., and Wang, D. C. (2014). Structural insights into the unique single-stranded DNA-binding mode of Helicobacter pylori DprA. Nucleic Acids Res. 42, 3478-3491. doi: 10.1093/nar/gkt1334

Wang, X., Liu, W., Zhu, D., Yang, L., Liu, M., Yin, S., et al. (2014). Comparative genomics of Riemerella anatipestifer reveals genetic diversity. BMC Genomics 15:479. doi: 10.1186/1471-2164-15-479

Xiong, A. S., Yao, Q. H., Peng, R. H., Duan, H., Li, X., Fan, H. Q., et al. (2006). PCR-based accurate synthesis of long DNA sequences. Nat. Protoc. 1, 791-797. doi: 10.1038/nprot.2006.103

Yadav, T., Carrasco, B., Hejna, J., Suzuki, Y., Takeyasu, K., and Alonso, J. C. (2013). Bacillus subtilis DprA recruits RecA onto single-stranded DNA and mediates annealing of complementary strands coated by SsbB and SsbA. J. Biol. Chem. 288, 22437-22450. doi: 10.1074/jbc.M113.478347

Zhang, X., Wang, M. S., Liu, M. F., Zhu, D. K., Biville, F., Jia, R. Y., et al. (2017). Contribution of RaeB, a putative RND-type transporter to aminoglycoside and detergent resistance in Riemerella anatipestifer. Front. Microbiol. 8:2435. doi: 10.3389/fmicb.2017.02435

Zhong, C. Y., Cheng, A. C., Wang, M. S., Zhu, D. K., Luo, Q. H., Zhong, C. D., et al. (2009). Antibiotic susceptibility of Riemerella anatipestifer field isolates. Avian Dis. 53, 601-607.

Zhu, D. K., Luo, H. Y., Liu, M. F., Zhao, X. X., Jia, R. Y., Chen, S., et al. (2018). Various profiles of tet genes addition to tet(X) in Riemerella anatipestifer isolates from ducks in China. Front. Microbiol. 9:585. doi: 10.3389/fmicb.2018.00585

Zhu, D. K., Yang, X. Q., Yang, H., Zhou, W. S., Song, X. H., Wang, J. B., et al. (2016). Comparative genomic analysis identifies structural features of CRISPRCas systems in Riemerella anatipestifer. BMC Genomics 17:689. doi: 10.1186/ s12864-016-3040-4

Conflict of Interest Statement: The authors declare that the research was conducted in the absence of any commercial or financial relationships that could be construed as a potential conflict of interest.

Copyright (c) 2019 Huang, Tian, Liu, Wang, Biville, Cheng, Zhu, Jia, Chen, Zhao, Yang, Wu, Zhang, Huang, Tian, Yu, Liu, Zhang, Pan, Rehman and Chen. This is an open-access article distributed under the terms of the Creative Commons Attribution License (CC BY). The use, distribution or reproduction in other forums is permitted, provided the original author(s) and the copyright owner(s) are credited and that the original publication in this journal is cited, in accordance with accepted academic practice. No use, distribution or reproduction is permitted which does not comply with these terms. 